\title{
AsYMPTOTIC EXPANSIONS OF THE COEFFICIENTS \\ IN ASYMPTOTIC SERIES SOLUTIONS \\ OF LINEAR DIFFERENTIAL EQUATIONS
}

\section{F. W. J. Olver}

ABSTRACT. In the neighborhood of an irregular singularity of rank one at infinity, a differential equation of the form

$$
\frac{d^{2} w}{d z^{2}}+f(z) \frac{d w}{d z}+g(z) w=0
$$

has well-known asymptotic solutions of the form

$$
e^{\lambda_{1} z} z^{\mu_{1}} \sum_{n=0}^{\infty} \frac{a_{n, 1}}{z^{n}}, \quad e^{\lambda_{2} z} z^{\mu_{2}} \sum_{n=0}^{\infty} \frac{a_{n, 2}}{z^{n}},
$$

in which $\lambda_{1}, \lambda_{2}, \mu_{1}$, and $\mu_{2}$ are constants. It is proved that for large $n$, the coefficients $a_{n, 1}$, and $a_{n, 2}$ can be expanded in asymptotic series of inverse factorials with explicit coefficients.

\section{Introduction}

It is well known that the Gamma function has an asymptotic expansion of the form

$$
\Gamma(n) \sim \sqrt{2 \pi} n^{n-(1 / 2)} e^{-n}\left\{1+\frac{1}{12 n}+\frac{1}{288 n^{2}}+\cdots\right\}
$$

as $n \rightarrow \infty$ in the sector $|\operatorname{ph} n| \leq \pi-\delta$, where $\delta$ is an arbitrary positive constant. Now suppose that $a$ and $b$ are fixed (or bounded) real or complex parameters. Then from (1.1) it follows that

$$
\frac{\Gamma(n+a) \Gamma(n+b)}{\Gamma(n+1)} \sim \sqrt{2 \pi} n^{n+a+b-(3 / 2)} e^{-n} \sum_{j=0}^{\infty} \frac{u_{j}}{n^{j}}
$$

in the same circumstances, where $u_{0}=1$ and higher coefficients are polynomials in $a$ and $b$. It is not difficult to find explicit expressions for the first few, for example,

$$
\begin{aligned}
u_{1}= & \frac{1}{2}\left(a^{2}+b^{2}-a-b\right)+\frac{1}{12} \\
u_{2}=\frac{1}{24}\{ & 3\left(a^{2}+b^{2}\right)^{2}-2\left(5 a^{3}+3 a^{2} b+3 a b^{2}+5 b^{3}\right) \\
& \left.\quad+2\left(5 a^{2}+3 a b+5 b^{2}\right)-3(a+b)\right\}+\frac{1}{288},
\end{aligned}
$$

Received December 18, 1992, revised May 12, 1993.

1991 Mathematics Subject Classification. Primary: 34E05; secondary: 33B15.

Key words and phrases. Asymptotic expansions, differential equations, exponential improvement, factorial series, Gamma function, Gevrey properties, irregular singularity.

This research was supported by the National Science Foundation under grants DMS 87-23039 and DMS 92-08690. 
but a general formula for $u_{j}$ is unavailable.

In consequence of Stirling's formula, the expansion (1.2) can be rearranged as a series of inverse factorials:

$$
\frac{\Gamma(n+a) \Gamma(n+b)}{\Gamma(n+1)} \sim \sum_{j=0}^{\infty} v_{j} \Gamma(n+a+b-j-1)
$$

again valid when $n \rightarrow \infty$ in $|\operatorname{ph} n| \leq \pi-\delta$. The interesting feature of this rearrangement is that in contrast to (1.2), the coefficients enjoy a simple general form given by

$$
v_{j}=(-)^{j} \frac{(1-a)_{j}(1-b)_{j}}{j !}
$$

Here we have employed Pochhammer's notation for ascending factorials, given by $(z)_{0}=1$ and

$$
(z)_{j}=z(z+1)(z+2) \cdots(z+j-1), \quad j \geq 1 .
$$

The results (1.3) and (1.4) are stated without proof by R. B. Dingle on p. 15 (and elsewhere) in [2].

An elegant proof of (1.4) has been supplied recently by R. B. Paris in the Appendix of [9]. Paris' proof is based on the identity

$$
\frac{\Gamma(n+a) \Gamma(n+b)}{\Gamma(n+1) \Gamma(n+a+b-1)}=\{1-\chi(n)\}_{2} F_{1}(1-a, 1-b ; 2-a-b-n ; 1)
$$

in which

$$
\chi(n)=\sin (\pi a) \sin (\pi b) \operatorname{cosec}(\pi n+\pi a) \operatorname{cosec}(\pi n+\pi b) .
$$

This result is valid when neither $n+a$ nor $n+b$ is a nonpositive integer. If we let $|\operatorname{Im} n| \rightarrow \infty$ with $\operatorname{Re} n$ fixed, then $\chi(n)$ becomes exponentially small and can be neglected. The coefficients in the required asymptotic expansion are then obtainable from the power-series expansion for the hypergeometric function in (1.5). 1

Paris needed the expansion (1.3) in the construction of exponentially-improved asymptotic expansions for the confluent hypergeometric function $U(a, a-b+1, z)$, with large $|z|$, via Mellin-Barnes integral representations. It was also needed by the present writer [7] in solving the same problem via the confluent hypergeometric differential equation.

The first purpose of the present paper is to supply an alternative, and quite simple, proof of (1.4). This proof serves as a preliminary for the main problem considered in the paper: the asymptotic nature of the coefficients in asymptotic series solutions of second-order linear differential equations in the neighborhood of an irregular singularity of unit rank. It transpires that the same method of proof yields some elegant, and apparently new, results for this more general problem.

\footnotetext{
${ }^{1}$ Another proof of (1.3) and (1.4) will be found in [8]. This proof is based on contour integration.
} 


\section{The Gamma-function ratio}

Let $n$ be a nonnegative integer, and for $n>-\operatorname{Re} a$ and $n>-\operatorname{Re} b$ define

$$
a_{n}=\frac{\Gamma(n+a) \Gamma(n+b)}{n !} .
$$

Then $a_{n}$ satisfies the recurrence relation

$$
n a_{n}=(n+a-1)(n+b-1) a_{n-1} .
$$

Motivated by (1.3), we substitute the following generalized asymptotic expansions into this relation

$$
\begin{aligned}
a_{n} & \sim \sum_{j=0}^{\infty} v_{j} \Gamma(n+a+b-j-1), \\
a_{n-1} & \sim \sum_{j=0}^{\infty} v_{j} \Gamma(n+a+b-j-2) .
\end{aligned}
$$

We seek to determine the unknown coefficients $v_{j}$. As a preliminary, we rearrange the coefficients in (2.2) as linear combinations of descending factorials; thus

$$
n=(n+a+b-j-1)+(j+1-a-b),
$$

and

$$
\begin{aligned}
(n+a-1)(n+b-1)=(n+ & a+b-j-1)(n+a+b-j-2) \\
& +(2 j+1-a-b)(n+a+b-j-2) \\
& +(j+1-a)(j+1-b) .
\end{aligned}
$$

On substituting (2.3) into (2.2), we derive

$$
\begin{aligned}
& \sum_{j=0}^{\infty} v_{j} \Gamma(n+a+b-j)+\sum_{j=0}^{\infty}(j+1-a-b) v_{j} \Gamma(n+a+b-j-1) \\
& \sim \sum_{j=0}^{\infty} v_{j} \Gamma(n+a+b-j)+\sum_{j=0}^{\infty}(2 j+1-a-b) v_{j} \Gamma(n+a+b-j-1) \\
& \quad+\sum_{j=0}^{\infty}(j+1-a)(j+1-b) v_{j} \Gamma(n+a+b-j-2) .
\end{aligned}
$$

Upon reduction, this asymptotic identity becomes

$$
\sum_{j=1}^{\infty} j v_{j} \Gamma(n+a+b-j-1) \sim-\sum_{j=1}^{\infty}(j-a)(j-b) v_{j-1} \Gamma(n+a+b-j-1),
$$

and, equating coefficients, we find that

$$
v_{j}=-\frac{(j-a)(j-b)}{j} v_{j-1}
$$

For the first coefficient we have, from (2.1) and (2.3),

$$
v_{0}=\lim _{n \rightarrow \infty} \frac{\Gamma(n+a) \Gamma(n+b)}{n ! \Gamma(n+a+b-1)}=1 .
$$


The required result (1.4) follows immediately. ${ }^{2}$

\section{Linear differential equations}

The asymptotic theory of the general homogeneous linear differential equation of the second order with an irregular singularity of rank one is given in [6], Chapter 7 . Without loss of generality we may suppose the singularity is at infinity. The main features of the theory are as follows. The differential equation has the form

$$
\frac{d^{2} w}{d z^{2}}+f(z) \frac{d w}{d z}+g(z) w=0
$$

in which the functions $f(z)$ and $g(z)$ can be expanded in power series

$$
f(z)=\sum_{s=0}^{\infty} \frac{f_{s}}{z^{s}}, \quad g(z)=\sum_{s=0}^{\infty} \frac{g_{s}}{z^{s}},
$$

that converge in a neighborhood of $z=\infty$. Furthermore, not all of the coefficients $f_{0}$, $g_{0}$, and $g_{1}$ vanish (otherwise, infinity would be a regular singularity). We may also suppose, without loss of generality, that

$$
f_{0}^{2} \neq 4 g_{0}
$$

cf. [6], Chapter $7, \S 1.3$. Let $\lambda_{1}$ and $\lambda_{2}$ be the roots of the characteristic equation

$$
\lambda^{2}+f_{0} \lambda+g_{0}=0 \text {. }
$$

In consequence of (3.3), we have $\lambda_{1} \neq \lambda_{2}$. Also define

$$
\mu_{1}=\frac{f_{1} \lambda_{1}+g_{1}}{\lambda_{2}-\lambda_{1}}, \quad \mu_{2}=\frac{f_{1} \lambda_{2}+g_{1}}{\lambda_{1}-\lambda_{2}} .
$$

Then (3.1) has unique solutions $w_{1}(z)$ and $w_{2}(z)$ such that

$$
w_{1}(z) \sim e^{\lambda_{1} z}\left\{\left(\lambda_{2}-\lambda_{1}\right) z\right\}^{\mu_{1}} \sum_{n=0}^{\infty} \frac{a_{n, 1}}{z^{n}}
$$

as $z \rightarrow \infty$ in the sector $\left|\operatorname{ph}\left(\lambda_{2}-\lambda_{1}\right) z\right| \leq \frac{3}{2} \pi-\delta$, and

$$
w_{2}(z) \sim e^{\lambda_{2} z}\left\{\left(\lambda_{2}-\lambda_{1}\right) z\right\}^{\mu_{2}} \sum_{n=0}^{\infty} \frac{a_{n, 2}}{z^{n}}
$$

as $z \rightarrow \infty$ in the sector $-\frac{1}{2} \pi+\delta \leq \operatorname{ph}\left(\lambda_{2}-\lambda_{1}\right) z \leq \frac{5}{2} \pi-\delta$. Here $\delta$ again denotes an arbitrary positive constant, and the coefficients are given by $a_{0,1}=a_{0,2}=1$ and, for $n \geq 1$,

$$
\begin{aligned}
& \left(\lambda_{1}-\lambda_{2}\right) n a_{n, 1}=\left(n-\mu_{1}\right)\left(n-1-\mu_{1}\right) a_{n-1,1} \\
& \quad+\sum_{s=1}^{n}\left\{\lambda_{1} f_{s+1}+g_{s+1}-\left(n-s-\mu_{1}\right) f_{s}\right\} a_{n-s, 1}
\end{aligned}
$$

\footnotetext{
${ }^{2}$ It is only the formula for $v_{j}$ that is at issue. By restricting $n$ to be a nonnegative integer in the present section, we do not impair the overall region of validity $|\mathrm{ph} n| \leq \pi-\delta$ of (1.3).
} 


$$
\begin{aligned}
& \left(\lambda_{2}-\lambda_{1}\right) n a_{n, 2}=\left(n-\mu_{2}\right)\left(n-1-\mu_{2}\right) a_{n-1,2} \\
& \quad+\sum_{s=1}^{n}\left\{\lambda_{2} f_{s+1}+g_{s+1}-\left(n-s-\mu_{2}\right) f_{s}\right\} a_{n-s, 2} .
\end{aligned}
$$

The main objective of this paper is to derive asymptotic expansions for $a_{n, 1}$ and $a_{n, 2}$ as $n \rightarrow \infty$. This type of problem has been studied before. Indeed, the asymptotic forms of the coefficients in formal series solutions of more general differential equations are well known; see [1], [3], [4], [10] and [11]. In the case of (3.1) itself, it is known how to construct asymptotic expansions for $a_{n, 1}$ and $a_{n, 2}$ in descending powers of $n$. However, the determination of the coefficients of the successive powers is cumbersome and no general formula is available. We shall show that by employing inverse factorial series instead, the coefficients can be expressed in simple explicit forms. It should be noted that Dingle [2, Chapters 13 and 24] also used inverse factorial series for expanding the late coefficients of asymptotic series. However, he did not consider the present problem (except in the special case mentioned in $\S 1$ ); furthermore, his analyses are of a formal character.

As in $\S 2$, our derivation of the new expansions for $a_{n, 1}$ and $a_{n, 2}$ is based directly on the recurrence relations satisfied by these coefficients and we employ only elementary analysis. An alternative, and somewhat shorter, proof based on some new integral representations for $a_{n, 1}$ and $a_{n, 2}$ will be found in [5]. An advantage of the present approach is that it is applicable to more general recurrence relations for which an integral representation of the solution may be unavailable.

\section{Main result}

Theorem 4.1. Let $J$ be an arbitrary nonnegative fixed integer. Then as $n \rightarrow \infty$

$$
\begin{array}{r}
a_{n, 1}=\frac{1}{\left(\lambda_{1}-\lambda_{2}\right)^{n}}\left[\Lambda_{1} \sum_{j=0}^{J-1} a_{j, 2}\left(\lambda_{1}-\lambda_{2}\right)^{j} \Gamma\left(n+\mu_{2}-\mu_{1}-j\right)\right. \\
\left.+O\left\{\Gamma\left(n+\mu_{2}-\mu_{1}-J\right)\right\}\right], \\
a_{n, 2}=\frac{1}{\left(\lambda_{2}-\lambda_{1}\right)^{n}}\left[\Lambda_{2} \sum_{j=0}^{J-1} a_{j, 1}\left(\lambda_{2}-\lambda_{1}\right)^{j} \Gamma\left(n+\mu_{1}-\mu_{2}-j\right)\right. \\
\left.+O\left\{\Gamma\left(n+\mu_{1}-\mu_{2}-J\right)\right\}\right],
\end{array}
$$

where $\Lambda_{1}$ and $\Lambda_{2}$ are constants.

This theorem is proved in the next two sections. At this stage we make the following observations:

(i) Actual values of the constants $\Lambda_{1}$ and $\Lambda_{2}$ are not derived in the present paper for the general case. ${ }^{3}$ However, in [5] it is shown that

$$
\Lambda_{1}=-i e^{\left(\mu_{2}-\mu_{1}\right) \pi i} C_{1} /(2 \pi), \quad \Lambda_{2}=i C_{2} /(2 \pi),
$$

\footnotetext{
${ }^{3}$ In [5] it is assumed that (3.1) has been normalized in such a way that $\lambda_{2}-\lambda_{1}=1$. This normalization is not assumed in the formulas given here.
} 
where $C_{1}, C_{2}$ are the coefficients in the connection formulas

$$
\begin{aligned}
& w_{1}(z)=e^{2 \pi i \mu_{1}} w_{1}\left(z e^{-2 \pi i}\right)+C_{1} w_{2}(z), \\
& w_{2}(z)=e^{-2 \pi i \mu_{2}} w_{2}\left(z e^{2 \pi i}\right)+C_{2} w_{1}(z) .
\end{aligned}
$$

See also [1], [3].

(ii) As in the case of (1.3), the expansions (4.1) and (4.2) are generalized asymptotic expansions. Numerically their character resembles that of ordinary Poincaré expansions, since the successive Gamma functions decrease asymptotically by a factor $n$.

\section{Proof of Theorem 4.1: The first approximation}

Our objective in this section is to prove that

$$
a_{n, 1}=\left(\lambda_{1}-\lambda_{2}\right)^{-n} \Gamma\left(n+\mu_{2}-\mu_{1}\right)\left\{\Lambda_{1}+O\left(n^{-1}\right)\right\}
$$

as $n \rightarrow \infty$. This result is a special case of known results; see, for example, [4]. However, for the sake of completeness and simplicity, we furnish a direct and elementary proof.

The underlying idea of the proof is as follows. In the recurrence relation (3.8), the first term on the right-hand side and the term in the sum for $s=1$ can be combined. In this way we obtain

$$
\begin{aligned}
\left(\lambda_{1}-\lambda_{2}\right) n a_{n, 1}=\left(n-1-c_{1}\right) & \left(n-1-c_{2}\right) a_{n-1,1} \\
& +\sum_{s=2}^{n}\left\{\lambda_{1} f_{s+1}+g_{s+1}-\left(n-s-\mu_{1}\right) f_{s}\right\} a_{n-s, 1},
\end{aligned}
$$

again valid when $n \geq 1 .{ }^{4}$ Here $c_{1}, c_{2}$ are constants that satisfy

$$
c_{1}+c_{2}=2 \mu_{1}+f_{1}-1, \quad c_{1} c_{2}=\mu_{1}\left(\mu_{1}+f_{1}-1\right)+\lambda_{1} f_{2}+g_{2} .
$$

We note that, in passing,

$$
\mu_{1}+\mu_{2}=-f_{1}
$$

(cf. (3.5)); in consequence, the first of (5.3) is equivalent to

$$
c_{1}+c_{2}=\mu_{1}-\mu_{2}-1 \text {. }
$$

If we neglect the sum on the right-hand side of (5.2), we obtain

$$
\left(\lambda_{1}-\lambda_{2}\right) n a_{n, 1}=\left(n-1-c_{1}\right)\left(n-1-c_{2}\right) a_{n-1,1} .
$$

Since $a_{0,1}=1$, this recurrence relation has the exact solution

$$
a_{n, 1}=\frac{1}{\Gamma\left(-c_{1}\right) \Gamma\left(-c_{2}\right)} \frac{\Gamma\left(n-c_{1}\right) \Gamma\left(n-c_{2}\right)}{\left(\lambda_{1}-\lambda_{2}\right)^{n} n !} .
$$

In consequence of (5.5) and Stirling's formula, the right-hand sides of (5.1) and (5.6) share the same asymptotic form; more precisely

$$
\frac{\Gamma\left(n-c_{1}\right) \Gamma\left(n-c_{2}\right)}{\left(\lambda_{1}-\lambda_{2}\right)^{n} n !}=\frac{\Gamma\left(n+\mu_{2}-\mu_{1}\right)}{\left(\lambda_{1}-\lambda_{2}\right)^{n}}\left\{1+O\left(n^{-1}\right)\right\}, \quad n \rightarrow \infty .
$$

\footnotetext{
${ }^{4}$ As usual, empty sums are interpreted as zero.
} 
Thus our essential object in this section is to show that the sum in (5.2) can be treated as a perturbation, whose effect is absorbable in the error term $O\left(n^{-1}\right)$ in (5.1).

Since the series (3.2) are assumed to converge in a neighborhood of $z=\infty$, there exist constants $F$ and $\rho$ such that

$$
\left|f_{s}\right| \leq F \rho^{s}, \quad\left|g_{s}\right| \leq F \rho^{s},
$$

for all $s$. Without loss of generality, we may suppose that $\rho \geq 1$. From these inequalities, it follows that for all positive values of the integer $n$ and all $s$ such that $1 \leq s \leq n$, we have

$$
\left|\lambda_{1} f_{s+1}+g_{s+1}-\left(n-s-\mu_{1}\right) f_{s}\right| \leq G(n+\rho) \rho^{s},
$$

where $G$ is another assignable constant.

Let $\gamma_{1}=\operatorname{Re} c_{1}, \gamma_{2}=\operatorname{Re} c_{2}$, and take $N$ to be a fixed positive integer that satisfies all of the following inequalities:

$$
N>\gamma_{1}, \quad N>\gamma_{2}, \quad \frac{\rho\left|\lambda_{1}-\lambda_{2}\right|(N+1)}{\left(N-\gamma_{1}\right)\left(N-\gamma_{2}\right)} \leq \frac{1}{2}
$$

and

$$
N \geq \sqrt{\left(1+\gamma_{1}\right)\left(1+\gamma_{2}\right)}-1 \quad \text { when } \quad\left(1+\gamma_{1}\right)\left(1+\gamma_{2}\right)>0 .
$$

(Obviously such an integer always exists.) When $n \geq N$, we substitute into (5.2) by means of the equation

$$
a_{n, 1}=\frac{\Gamma\left(n-c_{1}\right) \Gamma\left(n-c_{2}\right)}{\left(\lambda_{1}-\lambda_{2}\right)^{n} n !} \alpha_{n}
$$

This leads to the following recurrence relation for the quantities $\alpha_{n}$ :

$$
\alpha_{n}=\alpha_{n-1}+\sum_{s=2}^{n-N} h_{s}(n) \alpha_{n-s}+K(n), \quad n \geq N+1
$$

in which

$$
\begin{array}{r}
h_{s}(n)=\frac{\left\{\lambda_{1} f_{s+1}+g_{s+1}-\left(n-s-\mu_{1}\right) f_{s}\right\}\left(\lambda_{1}-\lambda_{2}\right)^{s-1}}{\left(n-1-c_{1}\right)\left(n-2-c_{1}\right) \cdots\left(n-s-c_{1}\right)} \\
\quad \times \frac{(n-1)(n-2) \cdots(n-s+1)}{\left(n-1-c_{2}\right)\left(n-2-c_{2}\right) \cdots\left(n-s-c_{2}\right)}
\end{array}
$$

and

$$
\begin{aligned}
K(n)= & \frac{\left(\lambda_{1}-\lambda_{2}\right)^{n-1}(n-1) !}{\Gamma\left(n-c_{1}\right) \Gamma\left(n-c_{2}\right)} \\
& \times \sum_{s=n-N+1}^{n}\left\{\lambda_{1} f_{s+1}+g_{s+1}-\left(n-s-\mu_{1}\right) f_{s}\right\} a_{n-s, 1} .
\end{aligned}
$$

In consequence of (5.9) and (5.10), we have

$$
\left|h_{s}(n)\right| \leq H_{s}(n), \quad 2 \leq s \leq n-N,
$$


where

$$
\begin{aligned}
H_{s}(n)=\frac{G(n+\rho) \rho^{s}\left|\lambda_{1}-\lambda_{2}\right|^{s-1}}{\left(n-1-\gamma_{1}\right)\left(n-2-\gamma_{1}\right) \cdots\left(n-s-\gamma_{1}\right)} \\
\quad \times \frac{(n-1)(n-2) \cdots(n-s+1)}{\left(n-1-\gamma_{2}\right)\left(n-2-\gamma_{2}\right) \cdots\left(n-s-\gamma_{2}\right)}
\end{aligned}
$$

We now introduce a majorizing sequence $\left\{\beta_{n}\right\}$ for $\left\{\alpha_{n}\right\}$, defined by

$$
\beta_{N}=\max \left(\left|\alpha_{N}\right|, 1\right)
$$

and

$$
\beta_{n}=\beta_{n-1}+\sum_{s=2}^{n-N} H_{s}(n) \beta_{n-s}+|K(n)|, \quad n \geq N+1
$$

Obviously

$$
\left|\alpha_{n}\right| \leq \beta_{n}, \quad n \geq N
$$

The next step is to prove that $\beta_{n}$ tends to a limit as $n \rightarrow \infty$. Suppose now that $n \geq N+3$. From the definition (5.17), we have

$$
\frac{H_{s+1}(n)}{H_{s}(n)}=\frac{\rho\left|\lambda_{1}-\lambda_{2}\right|(n-s)}{\left(n-s-1-\gamma_{1}\right)\left(n-s-1-\gamma_{2}\right)}, \quad 2 \leq s \leq n-N-1 .
$$

Let

$$
\phi(\nu)=\frac{\nu}{\left(\nu-1-\gamma_{1}\right)\left(\nu-1-\gamma_{2}\right)}, \quad \nu \geq N+1
$$

Then

$$
\frac{\phi^{\prime}(\nu)}{\phi(\nu)}=\frac{\left(1+\gamma_{1}\right)\left(1+\gamma_{2}\right)-\nu^{2}}{\nu\left(\nu-1-\gamma_{1}\right)\left(\nu-1-\gamma_{2}\right)} .
$$

In consequence of the assumed conditions (5.10), (5.11), we see that $\phi^{\prime}(\nu) \leq 0$ when $\nu \geq N+1$. Thus as $s$ increases the sequence of ratios $\left\{H_{s+1}(n) / H_{s}(n)\right\}$ is nondecreasing; accordingly

$$
\frac{H_{s+1}(n)}{H_{s}(n)} \leq \frac{H_{n-N}(n)}{H_{n-N-1}(n)}=\frac{\rho\left|\lambda_{1}-\lambda_{2}\right|(N+1)}{\left(N-\gamma_{1}\right)\left(N-\gamma_{2}\right)} \leq \frac{1}{2}
$$

cf. again (5.10). Hence

$$
H_{s}(n) \leq 2^{2-s} H_{2}(n), \quad 2 \leq s \leq n-N .
$$

From (5.19), it is obvious that the sequence $\beta_{N}, \beta_{N+1}, \beta_{N+2}, \ldots$ is increasing. With the aid of (5.18), (5.19), and (5.22) we derive

$$
\beta_{n}=\beta_{n-1}\left(1+\theta_{n}\right)
$$

where

$$
\left|\theta_{n}\right| \leq \sum_{s=2}^{n-N} H_{s}(n)+\frac{|K(n)|}{\beta_{n-1}} \leq 2 H_{2}(n)+|K(n)|
$$


From (5.17) with $s=2$, we see that $H_{2}(n)=O\left(n^{-2}\right)$ as $n \rightarrow \infty$. Furthermore, since the only unknown coefficients that appear in the sum in (5.15) are $a_{0,1}, a_{1,1}, \ldots, a_{N-1,1}$, it follows from Stirling's approximation and (5.9) that

$$
|K(n)|=\frac{\left(\left|\lambda_{1}-\lambda_{2}\right| e \rho\right)^{n}}{n^{n-\gamma_{1}-\gamma_{2}-(3 / 2)}} O(1), \quad n \rightarrow \infty .
$$

Thus $\theta_{n}=O\left(n^{-2}\right)$. In consequence, the infinite product

$$
\prod_{n=N+1}^{\infty}\left(1+\theta_{n}\right)
$$

converges, and so $\beta_{n}$ tends to a finite limit $\beta$, say, as $n \rightarrow \infty$.

Finally, from (5.13), (5.16), (5.20), and (5.22), we see that

$$
\left|\alpha_{n}-\alpha_{n-1}\right| \leq \beta \sum_{s=2}^{n-N} 2^{2-s} H_{2}(n)+|K(n)|=O\left(\frac{1}{n^{2}}\right),
$$

as before. Hence $\alpha_{n}$ approaches a finite limit $\Lambda_{1}$, say; furthermore

$$
\alpha_{n}=\Lambda_{1}+O\left(n^{-1}\right), \quad n \rightarrow \infty .
$$

In combination with (5.12) and (5.7), this establishes (5.1).

\section{Proof of Theorem 4.1: Higher approximations}

We now propose to extend the asymptotic approximation (5.1) into the desired asymptotic expansion (4.1). We first observe from (5.14) that in the relation (5.13), the functions $h_{s}(n)$ can be expanded in convergent series of the form

$$
h_{s}(n)=\frac{1}{n^{s}} \sum_{j=0}^{\infty} \frac{h_{j, s}}{n^{j}}
$$

when $n$ is large; the coefficients $h_{j, s}$ depend on $f_{s}, f_{s+1}, g_{s+1}, \lambda_{1}, \lambda_{2}, \mu_{1}, c_{1}$, and $c_{2}$.

Assume that $n \geq N+3$. Then we have

$$
\left|\sum_{s=3}^{n-N} h_{s}(n) \alpha_{n-s}+K(n)\right| \leq \beta \sum_{s=3}^{n-N} 2^{3-s} H_{3}(n)+|K(n)|=O\left(\frac{1}{n^{3}}\right) ;
$$

cf. (5.16), (5.17) (with $s=3),(5.21)$ and (5.23). Hence from (5.13) we have

$$
\alpha_{n}-\alpha_{n-1}=h_{2}(n) \alpha_{n-2}+O\left(\frac{1}{n^{3}}\right)=\frac{h_{0,2} \Lambda_{1}}{n^{2}}+O\left(\frac{1}{n^{3}}\right)
$$

cf. (5.25) and (6.1) (with $s=2$ ). Accordingly, from the expansion

$$
\alpha_{n}=\Lambda_{1}+\sum_{s=n}^{\infty}\left(\alpha_{s}-\alpha_{s+1}\right)
$$

we derive

$$
\alpha_{n}=\Lambda_{1}-\frac{h_{0,2} \Lambda_{1}}{n}+O\left(\frac{1}{n^{2}}\right), \quad n \rightarrow \infty
$$


Continuing the resubstitutions, we easily see that

$$
\alpha_{n} \sim \sum_{j=0}^{\infty} \frac{u_{j}}{n^{j}}
$$

where the coefficients $u_{j}$ are constants that depend on $\Lambda_{1}$, the coefficients in the expansions (3.2) for $f(z)$ and $g(z)$, and the Bernoulli numbers. ${ }^{5}$

In consequence, from (5.12) we have

$$
a_{n, 1} \sim \frac{\Gamma\left(n-c_{1}\right) \Gamma\left(n-c_{2}\right)}{\left(\lambda_{1}-\lambda_{2}\right)^{n} n !} \sum_{j=0}^{\infty} \frac{u_{j}}{n^{j}}, \quad n \rightarrow \infty .
$$

In turn, this expansion may be rearranged in the form

$$
a_{n, 1} \sim \frac{1}{\left(\lambda_{1}-\lambda_{2}\right)^{n}} \sum_{j=0}^{\infty} v_{j} \Gamma\left(n+\mu_{2}-\mu_{1}-j\right), \quad n \rightarrow \infty
$$

where $v_{0}, v_{1}, v_{2}, \ldots$ comprise another sequence of constants; cf. again (5.7).

In (6.3) it is clear that $v_{0}=u_{0}=\Lambda_{1}$. We shall find $v_{1}, v_{2}, \ldots$ by substituting into (3.8) by means of (6.3) and equating coefficients. We first need to overcome a difficulty stemming from the fact that the number of terms in the sum in (3.8) increases with $n$. This is achieved by truncating the sum at a fixed number of terms and estimating the remainder, as follows:

Lemma 6.1. If $M$ is any fixed nonnegative integer, then as $n \rightarrow \infty$

$$
\begin{aligned}
\sum_{s=M+1}^{n}\left\{\lambda_{1} f_{s+1}+g_{s+1}-\right. & \left.\left(n-s-\mu_{1}\right) f_{s}\right\} a_{n-s, 1} \\
= & O\left\{\frac{\Gamma\left(n+\mu_{2}-\mu_{1}-M\right)}{\left(\lambda_{1}-\lambda_{2}\right)^{n}}\right\} .
\end{aligned}
$$

Proof. Define $M_{0}$ to be the least nonnegative integer such that

$$
\operatorname{Re}\left(M_{0}+\mu_{2}-\mu_{1}\right)>0 \text { and }\left|M_{0}+\mu_{2}-\mu_{1}\right| \geq 2 \rho\left|\lambda_{1}-\lambda_{2}\right|,
$$

where $\rho$ is defined as in (5.8). From (5.1) it follows that

$$
\left|a_{s, 1}\right| \leq A\left|\left(\lambda_{1}-\lambda_{2}\right)^{-s} \Gamma\left(s+\mu_{2}-\mu_{1}\right)\right|, \quad s \geq M_{0},
$$

where $A$ is assignable independently of $s$.

From (5.9) and (6.6), we derive

$$
\left|\left\{\lambda_{1} f_{s+1}+g_{s+1}-\left(n-s-\mu_{1}\right) f_{s}\right\} a_{n-s, 1}\right| \leq K_{s}(n), \quad 1 \leq s \leq n-M_{0},
$$

where

$$
K_{s}(n)=A G(n+\rho) \rho^{s}\left|\left(\lambda_{1}-\lambda_{2}\right)^{s-n} \Gamma\left(n-s+\mu_{2}-\mu_{1}\right)\right| .
$$

When $1 \leq s \leq n-M_{0}-1$, we have

$$
\frac{K_{s+1}(n)}{K_{s}(n)}=\frac{\rho\left|\lambda_{1}-\lambda_{2}\right|}{\left|n-s+\mu_{2}-\mu_{1}-1\right|} \leq \frac{\rho\left|\lambda_{1}-\lambda_{2}\right|}{\left|M_{0}+\mu_{2}-\mu_{1}\right|} \leq \frac{1}{2}
$$

\footnotetext{
${ }^{5}$ Compare [6], p. 292, Example 3.2 .
} 
cf. (6.5). Hence

$$
\begin{aligned}
\sum_{s=M+1}^{n-M_{0}} \mid\left\{\lambda_{1} f_{s+1}+g_{s+1}-(n\right. & \left.\left.-s-\mu_{1}\right) f_{s}\right\} a_{n-s, 1} \mid \leq \sum_{s=M+1}^{n-M_{0}} 2^{M+1-s} K_{M+1}(n) \\
& \leq 2 K_{M+1}(n) \\
& =O\left\{\frac{\Gamma\left(n+\mu_{2}-\mu_{1}-M\right)}{\left(\lambda_{1}-\lambda_{2}\right)^{n}}\right\} .
\end{aligned}
$$

In the remaining part of the sum in (6.4), the only $a$ 's that appear are $a_{0,1}, a_{1,1}, \ldots$, $a_{M_{0}-1,1}$. With the aid, again, of (5.9), we see that

$$
\begin{aligned}
& \sum_{s=n-M_{0}+1}^{n} \mid\left\{\lambda_{1} f_{s+1}+\right.\left.g_{s+1}-\left(n-s-\mu_{1}\right) f_{s}\right\} a_{n-s, 1} \mid \\
&=O\left(n \rho^{n}\right)=O\left\{\frac{\Gamma\left(n+\mu_{2}-\mu_{1}-M\right)}{\left(\lambda_{1}-\lambda_{2}\right)^{n}}\right\}
\end{aligned}
$$

(cf. also (5.15) and (5.23).) Combination of (6.7) and (6.8) completes the proof of the lemma.

To continue the preparation of (3.8) for substitution by means of (6.3), we rearrange the coefficients as descending factorials (as in $\S 2$ ). Thus we set

$$
\begin{aligned}
n=(n+ & \left.\mu_{2}-\mu_{1}-j\right)+\left(\mu_{1}-\mu_{2}+j\right) \\
\left(n-\mu_{1}\right)\left(n-1-\mu_{1}\right)=(n+ & \left.\mu_{2}-\mu_{1}-j\right)\left(n+\mu_{2}-\mu_{1}-j-1\right) \\
& +2\left(j-\mu_{2}\right)\left(n+\mu_{2}-\mu_{1}-j-1\right) \\
& +\mu_{2}\left(\mu_{2}-1\right)+\left(1-2 \mu_{2}\right) j+j^{2}
\end{aligned}
$$

and

$\lambda_{1} f_{s+1}+g_{s+1}-\left(n-s-\mu_{1}\right) f_{s}=-f_{s}\left(n+\mu_{2}-\mu_{1}-j-s\right)+\left(\mu_{2}-j\right) f_{s}+\lambda_{1} f_{s+1}+g_{s+1}$.

We then find that

$$
\begin{aligned}
& \left(\lambda_{1}-\lambda_{2}\right)^{n-1}\left\{\left(\lambda_{1}-\lambda_{2}\right) n a_{n, 1}-\left(n-\mu_{1}\right)\left(n-1-\mu_{1}\right) a_{n-1,1}\right\} \\
& \sim \sum_{j=0}^{\infty}\left(\mu_{1}+\mu_{2}-j\right) v_{j} \Gamma\left(n+\mu_{2}-\mu_{1}-j\right) \\
& \quad-\sum_{j=0}^{\infty}\left\{\mu_{2}\left(\mu_{2}-1\right)+\left(1-2 \mu_{2}\right) j+j^{2}\right\} v_{j} \Gamma\left(n+\mu_{2}-\mu_{1}-j-1\right)
\end{aligned}
$$

and

$$
\begin{aligned}
& \left(\lambda_{1}-\lambda_{2}\right)^{n-1} \sum_{s=1}^{M}\left\{\lambda_{1} f_{s+1}+g_{s+1}-\left(n-s-\mu_{1}\right) f_{s}\right\} a_{n-s, 1} \\
& \sim-\sum_{s=1}^{M} f_{s}\left(\lambda_{1}-\lambda_{2}\right)^{s-1} \sum_{j=0}^{\infty} v_{j} \Gamma\left(n+\mu_{2}-\mu_{1}-j-s+1\right) \\
& \quad+\sum_{s=1}^{M}\left(\lambda_{1}-\lambda_{2}\right)^{s-1} \sum_{j=0}^{\infty}\left\{\left(\mu_{2}-j\right) f_{s}+\lambda_{1} f_{s+1}+g_{s+1}\right\} v_{j} \Gamma\left(n+\mu_{2}-\mu_{1}-j-s\right) .
\end{aligned}
$$


Bearing in mind Lemma 6.1, we may equate coefficients in the right members in (6.9) and (6.10) up to (but excluding) the terms in $\Gamma\left(n+\mu_{2}-\mu_{1}-M\right)$. From the leading terms, we derive

$$
\left(\mu_{1}+\mu_{2}\right) v_{0}=-f_{1} v_{0}
$$

in agreement with (5.4).

Next, on equating coefficients of $\Gamma\left(n+\mu_{2}-\mu_{1}-m\right)$, with $1 \leq m \leq M-1$, we have

$$
\begin{aligned}
\left(\mu_{1}+\mu_{2}-m\right) v_{m}- & \left\{\mu_{2}\left(\mu_{2}-1\right)+\left(1-2 \mu_{2}\right)(m-1)+(m-1)^{2}\right\} v_{m-1} \\
=- & -\sum_{s=1}^{m+1} f_{s}\left(\lambda_{1}-\lambda_{2}\right)^{s-1} v_{m-s+1} \\
& \quad+\sum_{s=1}^{m}\left\{\left(\mu_{2}-m+s\right) f_{s}+\lambda_{1} f_{s+1}+g_{s+1}\right\}\left(\lambda_{1}-\lambda_{2}\right)^{s-1} v_{m-s} .
\end{aligned}
$$

Upon rearrangement and use once again of (5.4), the last equation reduces to

$$
\begin{aligned}
& -m v_{m}=\left(m-\mu_{2}\right)\left(m-1-\mu_{2}\right) v_{m-1} \\
& \quad+\sum_{s=1}^{m}\left\{\lambda_{2} f_{s+1}+g_{s+1}-\left(m-s-\mu_{2}\right) f_{s}\right\}\left(\lambda_{1}-\lambda_{2}\right)^{s-1} v_{m-s} .
\end{aligned}
$$

From (3.9) it is easily verified that this is exactly the same as the recurrence relation satisfied by $\left(\lambda_{1}-\lambda_{2}\right)^{m} a_{m, 2}$. And since $a_{0,2}=1$, we conclude that

$$
v_{m}=\left(\lambda_{1}-\lambda_{2}\right)^{m} a_{m, 2} v_{0} .
$$

This result has been derived on the assumption that $m \leq M-1$, but since $M$ is arbitrary it becomes valid for all $m$. Substituting this result into (6.3), and recalling that $v_{0}=\Lambda_{1}$, we derive the required result (4.1).

The companion result (4.2) is proved in a similar manner, or we can appeal to symmetry. This completes the proof of Theorem 4.1 .

\section{Summary}

In $\S \S 1$ and 2 , we discussed the problem of constructing asymptotic expansions for a ratio of Gamma functions, and gave a new proof of the expansion

$$
\frac{\Gamma(n+a) \Gamma(n+b)}{\Gamma(n+1)} \sim \sum_{j=0}^{\infty}(-)^{j} \frac{(1-a)_{j}(1-b)_{j}}{j !} \Gamma(n+a+b-j-1)
$$

as $n \rightarrow \infty$ in $|\operatorname{ph} n| \leq \pi-\delta(<\pi)$.

In $\S \S 3$ to 6 , we used a similar method of proof to obtain new asymptotic expansions, again in series of inverse factorials, for the coefficients in asymptotic series solutions of homogeneous linear differential equations of the second order in the neighborhood of an irregular singularity of rank one. The results are summarized by Theorem 4.1.

It is easily verified that if Theorem 4.1 is applied to the following form of the confluent hypergeometric differential equation:

$$
\frac{d^{2} w}{d z^{2}}+\left(\frac{a-b+1}{z}-1\right) \frac{d w}{d z}-\frac{a}{z} w=0
$$

then the expansion (7.1) results. Thus (7.1) may be regarded as a special case of Theorem 4.1. Apart from obvious analytical and computational interests, the new 
expansions are of importance in the general theory of exponentially-improved asymptotic solutions of ordinary linear differential equations [5], just as the expansion (7.1) arises in the corresponding theory for the confluent hypergeometric equation [7], [9].

Acknowledgement. The writer is indebted to A. D. Wood for improvements in the presentation of this paper, and to the referees for helpful suggestions.

\section{References}

1. W. Balser, W. B. Jurkat and D. A. Lutz, On the reduction of connection problems for differential equations with an irregular singular point to ones with only regular singularities, II, SIAM J. Math. Anal. 19 (1988), 398-443.

2. R. B. Dingle, Asymptotic Expansions: Their Derivation and Interpretation, Academic Press, London, 1973.

3. G. K. Immink, $A$ note on the relationship between Stokes multipliers and formal solutions of analytic differential equations, SIAM J. Math. Anal. 21 (1990), 782-792.

4. W. Jurkat, D. Lutz and A. Peyerimhoff, Birkhoff invariants and effective calculations for meromorphic linear differential equations. I, J. Math. Anal. Appl. 53 (1976), 438-470.

5. A. B. Olde Daalhuis and F. W. J. Olver, Exponentially-improved asymptotic solutions of ordinary differential equations II: Irregular singularities of rank one, Proc. Roy. Soc. London, Ser. A, to appear.

6. F. W. J. Olver, Asymptotics and Special Functions, Academic Press, New York, 1974.

7. __ Exponentially-improved asymptotic solutions of ordinary differential equations I: The confluent hypergeometric function, SIAM J. Math. Anal. 24 (1993), 756-767.

8. - On an asymptotic expansion of a ratio of Gamma functions, manuscript.

9. R. B. Paris, Smoothing of the Stokes phenomenon using Mellin-Barnes integrals, J. Comput. Appl. Math. 41 (1992), 117-133.

10. J. P. Ramis, Les séries k-sommables et leur applications. In: Lecture Notes in Physics 126, (D. Iagolnitzer, ed.), Springer-Verlag, Berlin, 1980, 178-199.

11. Y. Sibuya, Gevrey property of formal solutions in a parameter. In: Asymptotic and Computational Analysis, (R. Wong, ed.), Lecture Notes in Pure and Applied Mathematics 124, Marcel Dekker, New York, 1990, 393-401.

Institute for Physical Science and Technology, University of Maryland, College Park, MARYLAND 20742, U. S. A. 